hep-ph/9803223

\title{
Cosmic Topological Defects, Highest Energy Cosmic Rays, and the Baryon Asymmetry of the Universe
}

\author{
Pijushpani Bhattacharjee \\ Laboratory for High Energy Astrophysics, Code 661, NASA/Goddard Space Flight Center, Greenbelt, MD 20771, USA. \\ and \\ Indian Institute of Astrophysics, Bangalore - 560 034, INDIA.
}

\begin{abstract}
It is pointed out that the observed extremely high energy cosmic rays (EHECR) above $10^{11} \mathrm{GeV}$ and the observed baryon asymmetry of the Universe (BAU), both may have a common origin in baryon number violating decays of supermassive " $\mathrm{X}$ " particles released from cosmic topological defects (TDs) such as cosmic strings and monopoles. The $\mathrm{X}$ particles produced by TDs in the recent epochs produce the EHECR, while the BAU is created by $\mathrm{X}$ particles released from TDs mainly in the very early Universe. In this scenario the EHECR is predicted to contain baryons as well as antibaryons with a small asymmetry between the two.
\end{abstract}

PACS numbers: $98.80 . \mathrm{Cq}, 98.70 . \mathrm{Sa}$

Cosmic Topological Defects (TDs) [1] 2] - magnetic monopoles, cosmic strings, domain walls, textures, superconducting cosmic strings, etc., as well as various hybrid systems consisting of these TDs - are predicted to form in the early Universe as a result of symmetry-breaking phase transitions envisaged in Grand Unified Theories (GUTs). The TDs can be thought of as 'constituted' of quanta of supermassive gauge and higgs fields (generically, "X" particles) of the underlying spontaneously broken gauge theory, with typical mass $m_{X} \lesssim 10^{16} \mathrm{GeV}$, the GUT symmetry breaking scale. TDs are topologically stable and so, once formed in the early Universe, they can survive forever with X particles 'trapped' inside them. However, from time to time, some TDs, through collapse, annihilation or other processes, can release the trapped X particles [3 11]. Decays of these X particles can give rise to extremely energetic nucleons, neutrinos and photons 12,13] with energies up to $\sim m_{X}$, which may potentially explain 14 18 the Extremely High Energy Cosmic Ray (EHECR) events with energies above $10^{11} \mathrm{GeV}$ [19].

The energies associated with the EHECR events are hard to obtain [14,20 within conventional scenarios of acceleration of charged particles in relativistic shocks associated with powerful astrophysical objects. In addition, there is the problem of absence of any obviously identifiable sources for the EHECR events [14,21]. These problems are avoided in the TD scenario in a natural way. Firstly, no acceleration mechanism is needed: The decay products of the $\mathrm{X}$ particles have energies up to $\sim m_{X}$ which can be as large as $\sim 10^{16} \mathrm{GeV}$. Secondly, the absence of obviously identifiable sources is not a problem because TDs need not necessarily be associated with any visible or otherwise active astrophysical objects.

The purpose of this Letter is to point out that if indeed decays of $\mathrm{X}$ particles from one or more TD-processes are responsible for the observed EHECR, then the same TD-processes may also be responsible for creating the Baryon Asymmetry of the Universe (BAU) due to CPand baryon number $(B)$ violating decays of the $\mathrm{X}$ particles. Production of X particles from TDs is an irreversible process, so the out-of-thermal-equilibrium condition necessary for the creation of BAU is (and hence the Sakharov conditions are) automatically satisfied.

The total baryon asymmetry produced by the decays of all X particles released from TDs at all epochs in the past is calculated below by normalizing the $\mathrm{X}$ particle production rate in the present epoch to that required to explain the EHECR flux. The result of this exercise is that the observed BAU can be obtained provided the temperature $T_{F}$, defined as the temperature below which any $B$-asymmetry produced by the $\mathrm{X}$ particle decays is not erased by other $B$-violating processes, is $\sim 10^{14} \mathrm{GeV}$.

Of course, as is well-known, $B$ violation through sphaleron transitions at high temperatures 22 could erase any net $B$-asymmetry created by other processes unless a non-vanishing value of $B-L(L$ being the Lepton number) is generated. This is a general problem for any scenario of so-called "GUT baryogenesis" in which BAU is generated through decay of supermassive (GUT-scale) $\mathrm{X}$ particles at high temperatures above the electroweak scale (few hundred GeV). The way to avoid this problem is also well-known: Assume a GUT like $S O(10)$, in which a net $B-L$ asymmetry may be generated through $L$-violating decays of certain Higgs particles (see, e.g., Refs. 23.24] for reviews of various baryogenesis scenar- 
ios); we shall assume this to be the case.

The possibility that $B$ violating decays of $\mathrm{X}$ particles released from TDs could be responsible for the BAU was first pointed out in two independent works [3, 4] in 1982, and subsequently studied further in Ref. 25. However, the possibility that TDs might be relevant for EHECR was not explored then. On the other hand, although there is currently much interest in the possibility that TDs may be responsible for EHECR, the possibility that the same TD processes might also have been responsible for the BAU seems to have escaped notice thus far. Thus, the present Letter explores the possible link between EHECR and BAU and a possible common origin of both in decays of $\mathrm{X}$ particles released from TDs.

The number density of $\mathrm{X}$ particles produced by TDs per unit time, $d n_{X} / d t$, can be generally written as 12

$$
\frac{d n_{X}}{d t}(t)=\frac{Q_{0}}{m_{X}}\left(\frac{t}{t_{0}}\right)^{-4+p}
$$

where $t_{0}$ denotes the present epoch, and $Q_{0}$ is the rate of energy density injected in the form of X particles in the present epoch. The quantity $Q_{0}$ and the parameter $p$ depend on the specific TD process under consideration. Processes with $p<1$ generally lead to unacceptably high rate of energy injection in the early cosmological epochs which would cause excessive ${ }^{4} \mathrm{He}$ photodisintegration and CMBR distortions [26] and are, therefore, unfavored in the context of EHECR. We shall, therefore, consider only the case $p=1$, which is representative of a number of TD processes studied so far [8 11] that are likely to be relevant for EHECR.

Although Eq. (1) is valid at all epochs after the formation of the relevant TDs in the early Universe, only those $\mathrm{X}$ particles produced in the relatively recent epochs and at relatively close-by, non-cosmological distances ( $\lesssim 100 \mathrm{Mpc}$ ) are relevant for the question of EHECR. This is because protons above $10^{11} \mathrm{GeV}$ produce pions in collision with the photons of the cosmic microwave background radiation (CMBR), and as a result suffer drastic energy loss (the so-called "GZK effect" 27]), which limits the source distance to effectively only a few tens of Mpc [14,21,28]. Distances of sources of photons of energies above $\sim 10^{11} \mathrm{GeV}$ are also similarly restricted due to absorption through $e^{+} e^{-}$production on the radio background photons (see, e.g., Refs. 13 18). The neutrinos can survive from much earlier cosmological epochs; however, the detected EHECR events are unlikely to be due to neutrinos because of their much lower interaction cross-section compared to those of nucleons and photons. Thus the EHECR can be produced, if at all, by X particles released from TDs only in the very recent epochs.

The BAU, on the other hand, must already have been in place in the early Universe; indeed, in order not to disturb the successful predictions of the primordial nucleosynthesis scenario, the bulk of the BAU must have been created before a temperature of $\sim 0.1 \mathrm{MeV}$, and probably well before the quark-hadron phase transition at a temperature of few hundred $\mathrm{MeV}$. This is naturally achieved in the TD scenario because, the $\mathrm{X}$ particle production rate being $\propto t^{-3}$, the bulk of the contribution to the BAU comes from production and decay of X particles at the earliest possible epoch characterized by the temperature $T_{F}$ mentioned earlier.

The $\mathrm{X}$ particles (including $\bar{X}$ 's) released from TDs decay typically into quarks and leptons (and/or their antiparticles). The quarks "fragment" into jets of hadrons - mostly pions, with a small admixture (typically $\lesssim$ $10 \%$ ) of baryons and antibaryons (nucleons and antinucleons). Thus photons and neutrinos resulting from the decays of neutral and charged pions, respectively, dominate the total particle yield at production [29], while the baryon asymmetry associated with the $\mathrm{X}$-decay is contained in the relatively small baryon yield.

The hadronic spectra should be similar to those measured for jets seen in $e^{+} e^{-}$annihilation experiments, which are well described by QCD [30]. For the energy regions of our interest, the nucleon, photon and neutrino spectra resulting from the decay of each $\mathrm{X}$ particle can be approximated by power-laws in energy $\left(\propto E^{-\alpha}\right)$ with, typically, $1.3 \lesssim \alpha \lesssim 1.7$.

We can now make a rough estimate of $Q_{0}^{\mathrm{EHECR}}$, the value of $Q_{0}$ in Eq. (11) required to explain the observed EHECR flux. We can do this by normalizing the predicted photon flux with the observed EHECR flux since, in our scenario, photons dominate the observable particle flux at EHECR energies. Let us assume a typical $B$-violating decay mode of the $\mathrm{X}$ into a quark and a lepton: $X \rightarrow q \ell$. The quark produces a hadronic jet. The photons from the decay of neutral pions in the jet carry a total energy $E_{\gamma, \text { Total }} \simeq\left(\frac{1}{3} \times 0.9 \times \frac{1}{2}\right) m_{X}=0.15 m_{X}$, where we have assumed that on average $\sim 90 \%$ of a jet's total energy is carried by pions. Assuming a power-law photon spectrum with index 1.5 , the photon injection spectrum due to a single $\mathrm{X}$ particle decay can be written as $\frac{d N_{\gamma}}{d E_{\gamma}}=\frac{1}{m_{X}} \times 0.3\left(\frac{2 E_{\gamma}}{m_{X}}\right)^{-1.5}$, which is properly normalized with the total photon energy $E_{\gamma, \text { Total }}$. We can neglect cosmological evolution effects and take the present epoch values of the relevant quantities, since photons of EHECR energies have a cosmologically negligible absorption length of only $\sim$ few tens of Mpc. With these assumptions, the photon flux $j_{\gamma}\left(E_{\gamma}\right)$ is simply given by $j_{\gamma}\left(E_{\gamma}\right) \simeq \frac{1}{4 \pi} \lambda\left(E_{\gamma}\right) \frac{d n_{X}}{d t} \frac{d N_{\gamma}}{d E_{\gamma}}$, where $\lambda\left(E_{\gamma}\right)$ is the pair production absorption path length of a photon of energy $E_{\gamma}$.

Noting that $d n_{X} / d t=Q_{0} / m_{X}$, and normalizing the above flux to the measured EHECR flux corresponding to the highest energy event at $\sim 3 \times 10^{11} \mathrm{GeV}$, given by $j\left(3 \times 10^{11} \mathrm{GeV}\right) \approx 5.6 \times 10^{-41} \mathrm{~cm}^{-2} \mathrm{eV}^{-1} \mathrm{sec}^{-1} \mathrm{sr}^{-1}$ [19], we get 
$Q_{0}^{\mathrm{EHECR}} \approx 1.2 \times 10^{-30} \frac{\mathrm{GeV}}{\mathrm{cm}^{3} \mathrm{sec}}\left(\frac{30 \mathrm{Mpc}}{\lambda_{\gamma, 300}}\right)\left(\frac{m_{X}}{10^{16} \mathrm{GeV}}\right)^{\frac{1}{2}}$,

where $\lambda_{\gamma, 300}$ is the absorption path length of a $300 \mathrm{EeV}$ photon $\left(1 \mathrm{EeV} \equiv 10^{18} \mathrm{eV}\right)$. The subscript 0 stands for the present epoch. More detailed numerical calculations of the predicted EHECR flux have been done [16] by solving the relevant full transport equations, which yield a similar number for $Q_{0}^{\mathrm{EHECR}}$ as derived above.

Eq. (2) implies a required $\mathrm{X}$ particle production rate $\dot{n}_{X, 0} \sim 1.1 \times 10^{35}\left(\frac{30 \mathrm{Mpc}}{\lambda_{\gamma, 300}}\right)\left(\frac{m_{X}}{10^{16} \mathrm{GeV}}\right)^{-1 / 2} \mathrm{Mpc}^{-3} \mathrm{yr}^{-1}$. In addition, in order that the resulting EHECR flux be not too anisotropic, there must be several TD sources of these X particles today within a typical "GZK" volume of radius $\sim 30 \mathrm{Mpc}$. This puts rather stringent constraints on the possible types of TD sources [18]. Currently, the most viable TDs in this regard seem to be collapsing monopolonia [5,9] and "necklace" 10], although X particle production due to repeatedly self-intersecting kinky cosmic string loops [31] also remains as a possibility. Empirically, in terms of the number densities of TDs, perhaps the most well-constrained are the monopoles [23]. Noting that a GUT monopole typically has a mass $m_{M} \sim 40 m_{X}$, so that each monopolonium collapse releases $\sim 80 \mathrm{X}$ particles, the above estimate of required $\dot{n}_{X, 0}$ implies the condition $\left(n_{M \bar{M}}^{c} / n_{M}\right) \Omega_{M} h^{2} \simeq 1.2 \times$ $10^{-8}\left(\frac{30 \mathrm{Mpc}}{\lambda_{\gamma, 300}}\right)\left(\frac{m_{X}}{10^{16} \mathrm{GeV}}\right)^{1 / 2}\left(\Omega_{0} h^{2}\right)^{-1 / 2}$, where $n_{M \bar{M}}^{c}$ is the number density of monopolonia in the final stage of collapse in the present epoch, i.e., the ones that are currently, and will be (over the next one Hubble time), disappearing due to $M \bar{M}$ annihilation [32], $n_{M}$ is the number density of monopoles (including antimonopoles), $\Omega_{M}$ is the mass density contributed by monopoles and $\Omega_{0}$ the total mass density (both in units of the critical mass density of closure of the Universe), and $h$ is the present Hubble constant in units of $100 \mathrm{~km} \mathrm{sec}^{-1} \mathrm{Mpc}^{-1}$. An equilibrium Saha ionization formalism [5.9] for the monopolonium formation process indicates that the above requirement on $\left(n_{M \bar{M}}^{c} / n_{M}\right)$ is consistent with the known independent upper limits on the monopole abundance such as the closure limit $\Omega_{M} h^{2} \leq 1$, and also with the more stringent "Parkar limit" [23] $\left(\Omega_{M} h^{2}\right)_{\text {Parkar }} \lesssim$ $4 \times 10^{-3}\left(m_{M} / 10^{16} \mathrm{GeV}\right)^{2}$. For a recent discussion of phenomenological aspects of various TD sources of EHECR, see Ref. [18].

Now, taking $Q_{0}=Q_{0}^{\text {EHECR }}$ in Eq. (11) with $p=1$, the total BAU produced by $\mathrm{X}$ particle decays is simply given by [3]

$$
\frac{n_{B}}{s} \simeq \Delta B \int_{t_{F}}^{t_{0}} \frac{d t}{s} \frac{d n_{X}}{d t}(t)=\Delta B \frac{Q_{0}^{\mathrm{EHECR}}}{m_{X}} \int_{t_{F}}^{t_{0}} \frac{d t}{s}\left(\frac{t_{0}}{t}\right)^{3}
$$

where $t_{F}$ is the "freeze-out" time corresponding to the temperature $T_{F}$ mentioned earlier, $\Delta B$ is the mean net baryon number produced in the decay of an X particle, and $s=\left(2 \pi^{2} / 45\right) g_{* S} T^{3}$ is the entropy density at temperature $T$ (corresponding to time $t$ ), $g_{* S} \sim 100$ being the relevant number of relativistic degrees of freedom [23]. In writing Eq. (3), we have assumed that the entropy produced in the decays of $\mathrm{X}$ particles themselves at any time $t$ in the early epochs of our interest is negligible compared to the ambient entropy density. This is a good approximation [3] in our scenario because the $\mathrm{X}$ particles do not dominate the density of the Universe at any time.

Using the standard time-temperature relation in the early Universe [23], we see that the integral in Eq. (3) is dominated by the contribution at the earliest time $t_{F}$, giving

$$
\begin{gathered}
\frac{n_{B}}{s} \simeq 2.2 \times 10^{-9}\left(\Delta B / 10^{-2}\right)\left(m_{X} / 10^{16} \mathrm{GeV}\right)^{-1 / 2} \\
\left(T_{F} / 10^{16} \mathrm{GeV}\right) \Omega_{0}^{-3 / 2}(h / 0.65)^{-3},
\end{gathered}
$$

where we have used $\lambda_{\gamma, 300}=30 \mathrm{Mpc}$ in Eq. (2).

In a general GUT-baryogenesis scenario, the temperature $T_{F}$ is $\sim m_{X}$ 23.24. We thus see that, depending on the values of $\Delta B, h$ and $\Omega_{0}$, the estimated BAU of $\sim(4$ -14) $\times 10^{-11}$ [23 can be obtained with $m_{X} \sim 10^{14} \mathrm{GeV}$. (Note that $m_{X}$ much above $10^{14} \mathrm{GeV}$ tends to overproduce BAU, as well as the EHECR [17], and may, therefore, be unfavored.)

There are many uncertainties in the above estimates. An accurate estimate can be obtained only through detailed numerical calculations involving solution of the relevant Boltzmann equation that incorporates all Bviolating interactions, not just the decays of X's. Nevertheless, the rough estimate of BAU made above should give us an idea of the kind of numbers to expect.

Recently, attempts have been made [33] to revive the "standard" GUT baryogenesis through decay of massive $\mathrm{X}$ particles produced during "preheating" stage in the inflationary Universe. While these X particles may produce the BAU, they cannot be responsible for the EHECR because these $\mathrm{X}$ particles all decayed away in the early Universe and are not produced in the recent epochs. On the other hand, it has been suggested [34] that massive stable particles (with life-time $\gtrsim$ age of the Universe) may be created in the early Universe through vacuum fluctuations during inflation, which can act as the dark matter, and a small fraction of those decaying in the present epoch may give rise to the EHECR. However, in this scenario, the BAU (which must have been created in the early Universe) cannot have an origin in decays of these stable X particles, which decay, if at all, only in the recent epochs. In contrast, in the TD scenario outlined above, the $\mathrm{X}$ particles themselves are unstable, but they are produced continually at all epochs including the recent epochs, so that both BAU as well as EHECR can be produced. 
Note that, in the TD scenario, the EHECR at production is predicted to contain baryons as well as antibaryons with a small asymmetry between the two. It remains, however, as a challenge at this stage to devise a scheme that would enable one to distinguish EHECR air-showers initiated by protons from those initiated by antiprotons, thereby to test the prediction experimentally.

To summarize, then, not only the extremely high energy cosmic rays, but the entire 'low' energy baryonic content of the Universe may, at some stage or other, have originated from topological defects. Thus the BAU may be a dynamically evolving quantity, and the EHECR observed today may represent the baryon creation process itself "in action" in the Universe today.

I wish to thank Q. Shafi, F. Stecker, R. Streitmatter, and G. Yodh for discussions. This work is supported by NAS/NRC and NASA.

[1] T.W.B. Kibble, J. Phys. A9, 1387 (1976).

[2] A. Vilenkin and E.P.S. Shellard, Cosmic Strings and other Topological Defects (Cambridge Univ. Press, Cambridge, 1994); M. Hindmarsh and T. W. B. Kibble, Rep. Prog. Phys. 58, 477 (1995); T. Vachaspati, Topological Defects in the Cosmos and Lab hep-ph/9802311) (to appear in Contemporary Physics.

[3] P. Bhattacharjee, T.W.B. Kibble, and N. Turok, Phys. Lett. B 119, 95 (1982).

[4] S. Nussinov, Phys. Lett. B 110, 221 (1982).

[5] C.T. Hill, Nucl. Phys. B 224, 469 (1983).

[6] C.T. Hill, D.N. Schramm, and T.P. Walker, Phys. Rev. D 36, 1007 (1987).

[7] J. H. MacGibbon and R. H. Brandenberger, Nucl. Phys. B 331, 153 (1990); P. Bhattacharjee, Phys. Rev. D 40, 3968 (1989); M. Mohazzab and R. Brandenberger, Int. Jour. Mod. Phys. D 2, 183 (1993).

[8] P. Bhattacharjee, in Astrophysical Aspects of the Most Energetic Cosmic Rays, eds. M. Nagano and F. Takahara (World Scientific, Singapore, 1991), pp. 382 - 399; P. Bhattacharjee and N. C. Rana, Phys. Lett. B 246, 365 (1990); A. J. Gill and T. W. B. Kibble, Phys. Rev. D 50, 3660 (1994).

[9] P. Bhattacharjee and G. Sigl, Phys. Rev. D 51, 4079 (1995).

[10] V. Berezinsky and A. Vilenkin, Phys. Rev. Lett. 79, 5202 (1997).

[11] G. Vincent, N.D. Antunes, and M. Hindmarsh, Phys. Rev. Lett. 80, 2277 (1998); G. R. Vincent, M. Hindmarsh, and M. Sakellariadou, Phys. Rev. D 56, 637 (1997).

[12] P. Bhattacharjee, C.T. Hill, and D.N. Schramm, Phys. Rev. Lett. 69, 567 (1992).

[13] F.A. Aharonian, P. Bhattacharjee, and D.N. Schramm, Phys. Rev. D 46, 4188 (1992).

[14] G. Sigl, D.N. Schramm, and P. Bhattacharjee, Astropart.
Phys. 2, 401 (1994).

[15] G. Sigl, S. Lee, D. N. Schramm, and P. Bhattacharjee, Science, 270, 1977 (1995).

[16] G. Sigl, S. Lee, and P. Coppi, astro-ph/9604093; R. J. Protheroe and T. Stanev, Phys. Rev. Lett. 77, 3708 (1996); 78, 3420 (1997) (E); G. Sigl, S. Lee, D. N. Schramm, and P. Coppi, Phys. Lett. B 392, 129 (1997).

[17] P. Bhattacharjee, Q. Shafi, and F. W. Stecker, Phys. Rev. Lett. 80, 3698 (1998).

[18] V. Berezinsky, P. Blasi, and A. Vilenkin, astro$\mathrm{ph} / 9803271$.

[19] D.J. Bird et al, Phys. Rev. Lett. 71, 3401 (1993); Astrophys. J. 441, 144 (1995); N. Hayashida et al, Phys. Rev. Lett. 73, 3491 (1994); S. Yoshida et al, Astropart. Phys. 3, 105 (1995).

[20] A. M. Hillas, Ann. Rev. Astron. Astrophys. 22, 425 (1984); C. A. Norman, D. B. Melrose, and A. Achterberg, Astrophys. J. 454, 60 (1995).

[21] J. W. Elbert and P. Sommers, Astrophys. J. 441, 151 (1995).

[22] V.A. Kuzmin, V.A. Rubakov, and M. E. Shaposhnikov, Phys. Lett. B 155, 36 (1985).

[23] E.W. Kolb and M.S. Turner, The Early Universe (Addison-Wesley, Redwood City, California, 1990).

[24] A.D. Dolgov, Phys. Rep. 222, 309 (1992); hep$\mathrm{ph} / 9707419$.

[25] R. Brandenberger, A-C. Davis, and M. Hindmarsh, Phys. Lett. B 263, 239 (1991).

[26] G. Sigl, K. Jedamzik, D. N. Schramm, and V. Berezinsky, Phys. Rev. D 52, 6682 (1995).

[27] K. Greisen, Phys. Rev. Lett. 16, 748 (1966); G. T. Zatsepin and V. A. Kuzmin, Pisma Zh. Eksp. Teor. Fiz. 4, 114 (1966) [ JETP. Lett. 4, 78 (1966)]; F. W. Stecker, Phys. Rev. Lett. 21, 1016 (1968).

[28] F. A. Aharonian and J. W. Cronin, Phys. Rev. D 50, 1892 (1994).

[29] However, protons may still dominate over photons in the observed particle flux at energies $\lesssim 10^{11} \mathrm{GeV}$ because of the larger attenuation length of protons compared to the absorption length of photons at these energies, while the photons can dominate over protons above a few times $10^{11} \mathrm{GeV}$ (see Refs. 13, 18).

[30] Yu. L. Dokshitzer, V. A. Khoze, A. H. Mueller, and S. I. Troyan, Basics of perturbative QCD (Editions Frontiers, Saclay, 1991); V. A. Khoze and W. Ochs, Int. J. Mod. Phys. A12, 2949 (1997).

[31] X. A. Siemens and T. W. B. Kibble, Nucl. Phys. B 438, 307 (1995).

[32] These monopolonia were formed over a Hubble time roughly at about the epoch of primordial nucleosynthesis [9].

[33] E. W. Kolb, A. Linde, and A. Riotto, Phys. Rev. Lett. 77, 4290 (1996); E. W. Kolb, A. Riotto, and I. Tkachev, Phys. Lett. B 423, 348 (1998).

[34] V. A. Kuzmin and V. A. Rubakov, astro-ph/9709187; V. Berezinsky, M. Kachelriess and A. Vilenkin, Phys. Rev. Lett. 79, 4302 (1997); V. Kuzmin and I. Tkachev, hep-ph/9802304; D. Chung, E. W. Kolb, and A. Riotto, hep-ph/9802238. 